\title{
G. GAMARRA
}

\section{Calidad de vida en ancianos tratados con diálisis crónica}

\section{Quality of life in elderly patients treated with chronic dialysis}

En los Estados Unidos de América, según lo reportado en el registro, el tratamiento dialítico más utilizado en personas mayores de 75 años de edad, con enfermedad renal estadio 5 es la hemodiálisis, realizada en un centro de diálisis en el $96 \%$ de los casos; menos del $1 \%$ son tratados con hemodiálisis en casa y poco más del $3 \%$ reciben diálisis peritoneal continua ambulatoria (CAPD) o diálisis peritoneal con máquina cicladora (CCPD) (1).

En Colombia, como en la mayoría de los países del mundo, se ha observado un aumento en la edad promedio de los pacientes que acceden a las diferentes modalidades de terapia dialítica y no es inusual encontrar personas mayores de 80 años recibiendo alguna de las modalidades de tratamiento, sin que existan adecuados estudios que demuestren un efecto benéfico evidenciado no solo en mayor sobrevida sino en una mejor calidad de vida y menor morbilidad.

En el presente número de Acta Médica Colombiana se publica un artículo realizado por Martínez y cols. titulado "Calidad de vida y estado funcional de ancianos con enfermedad renal crónica estadio 5 en terapia dialítica" cuyo objetivo fue evaluar la calidad de vida, estado funcional, aspectos cognitivos $\mathrm{y}$ afectivos en pacientes mayores de 65 años con enfermedad renal crónica estadio 5 en terapia de reemplazo renal en una unidad de diálisis de la ciudad de Manizales (2).

Aunque no era el objetivo, los autores realizan comparaciones entre dos grupos de pacientes adultos mayores en terapia dialítica, uno que recibe diálisis peritoneal y el otro, que recibe hemodiálisis. Se comparan las características socio-demográficas, comorbilidades, deterioro cognitivo, depresión, capacidad funcional, fragilidad y calidad de vida. Es evidente, que en el mencionado estudio, no se pretendía comparar el efecto que producen las dos modalidades de terapia de diálisis utilizadas sobre las diferentes variables estudiadas, lo que habría sido deseable, pero se habría requerido un diseño metodológico diferente.
La decisión sobre el tipo de diálisis utilizado en cada paciente obedece a diversos factores, lo que genera de entrada, un sesgo en la selección de los sujetos del estudio; existen preferencias del paciente sobre uno $\mathrm{u}$ otro tratamiento que favorecen su adherencia, como también hay aspectos que influyen en la decisión del médico sobre la indicación de la terapia, relacionados con las particularidades de cada paciente. Adicionalmente, en Colombia existen otros factores que determinan la asignación a la terapia dialítica y son los relacionados con las políticas establecidas por las Empresas Promotoras de Salud (EPS) y por las empresas proveedoras de servicios de diálisis. Para evitar el sesgo en la selección habría que partir de una situación, que obviamente no ocurre en la vida real, en la que los pacientes estudiados tuvieran la misma probabilidad de ser asignados a cualquiera de las dos modalidades de diálisis.

En el estudio citado, y publicado en este número de la revista, al tener un diseño de corte transversal, en el que las diferentes mediciones se realizan en un mismo momento del tiempo, no es posible establecer asociaciones causales, ya que los dos eventos se encuentran presentes cuando se efectúa su medición; por ejemplo: actividad física y depresión pueden encontrarse de manera simultánea en el mismo paciente y no es posible determinar si una de ellas favorece la aparición de la otra; la depresión reduce la actividad física o la reducción de la actividad física favorece la aparición de depresión?. A pesar de las pruebas estadísticas utilizadas, en particular del uso de la regresión logística, con estudios de corte transversal no se puede pretender hacer predicciones. Las limitaciones del estudio no solamente se deben al inadecuado tamaño de la muestra, que no fue estimado con base en una hipótesis, sino de manera importante, al diseño utilizado y a los sesgos que se presentan; por tales motivos, los resultados encontrados solo pueden ser aplicados a los sujetos participantes del estudio y en ningún caso es posible tratar
Ver artículo: página 13

Dr. Germán Gamarra Hernández: Especialista en Medicina Interna y Nefrología. Magíster en Epidemiología Clínica. Secretario General, Academia Nacional de Medicina de Colombia. Bogotá D.C. (Colombia). Correspondencia: Dr. Germán Gamarra. Bogotá D.C. (Colombia). E-mail: ggamarra2000@yahoo.com 
de generalizar a otros pacientes. El diseño adecuado para el estudio propuesto debería haber sido prospectivo y de cohortes, en donde mediante un seguimiento longitudinal de los pacientes, se observe la exposición y la aparición del desenlace o los desenlaces posteriores.

En los pacientes ancianos o adultos mayores se presentan diversas condiciones psicosociales y comorbilidades que afectan la sobrevida y la calidad de vida; los resultados encontrados en algunos estudios presentan conclusiones diferentes debido al tipo de paciente, período de evaluación, diseño del estudio y desenlace medido $(3,4)$. Hay estudios observacionales que han mostrado una mejoría en la sobrevida en pacientes ancianos tratados con diálisis en comparación con aquellos tratados de manera conservadora, en particular en aquellos pacientes que tienen menor comorbilidad cardiovascular; por otra parte, se ha sugerido por algunos autores, que los adultos mayores que asisten de manera regular a la terapia en centros de hemodiálisis, pueden mejorar su calidad de vida como consecuencia del estímulo social que genera la interacción con personas más jóvenes.

La comparación de los desenlaces cuando se utilizan las diferentes modalidades de diálisis, en particular peritoneal versus hemodiálisis tiene múltiples problemas; los más importantes, ya fueron anotados y se relacionan con los factores que intervienen en la selección del paciente; adicionalmente, y no menos importante es que con frecuencia un paciente puede alternar diferentes formas de terapia de remplazo renal, incluyendo el trasplante; durante cada período de tratamiento, las circunstancias individuales pueden variar, por la modificación de las condiciones comórbidas o por el deterioro producido por la edad y la progresión de la enfermedad renal.

Hay otros estudios, aunque escasos, que evalúan la mortalidad relacionada con las diferentes formas de diálisis en adultos mayores; los resultados han sido contradictorios, especialmente por varias de las razones, relacionadas con las características demográficas o la comorbilidad que presentaron los pacientes (5).

El mayor acceso de pacientes con edad avanzada a la terapia de diálisis hace necesario realizar estudios, metodológicamente rigurosos y con el diseño adecuado, que permitan evaluar los efectos derivados de la misma en esta población y conocer los verdaderos beneficios que se logran con el tratamiento. Es importante desarrollar investigación clínica en esta área, máximo si se tiene en cuenta el impacto económico que la atención del enfermo renal y en particular de las personas en edad avanzada, produce sobre el sistema de salud.

\section{Referencias}

1. Excerpts from the USRDS 2005 annual data report: Atlas of end-stage renal disease in the United States. Am J Kidney Dis 2006; 47 (Suppl 1):S1.

2. Martínez HL, Restrepo CA, Arango F. Calidad de vida y estado funcional de ancianos con enfermedad renal crónica estadio 5 en terapia dialítica. Acta Med Colomb 2015: 40: 13-19.

3. Kurella Tamura M, Covinsky KE, Chertow GM, et al. Functional status of elderly adults before and after initiation of dialysis. N Engl J Med 2009; 361:1539.

4. Carson RC, Juszczak M, Davenport A, Burns A. Is maximum conservative management an equivalent treatment option to dialysis for elderly patients with significant comorbid disease? Clin J Am Soc Nephrol 2009; 4:1611.

5. Jaar BG, Coresh J, Plantinga LC, et al. Comparing the risk for death with peritoneal dialysis and hemodialysis in a national cohort of patients with chronic kidney disease. Ann Intern Med 2005; 143:174. 